\title{
Association of Body Mass Index with Suicide Behaviors, Perceived Stress, and Life Dissatisfaction in the Korean General Population
}

\author{
Haesoo Kim 1 , Hong Jin Jeon ${ }^{2}$, Jae Nam Bae ${ }^{3}$, Maeng Je Cho ${ }^{4}$, \\ Seong Jin $\mathrm{Cho}^{5}$, Hyochul Lee ${ }^{1}$, and Jin Pyo Hong ${ }^{2}$ \\ ${ }^{1}$ Department of Psychiatry, Samsung Medical Center, Seoul, Republic of Korea \\ ${ }^{2}$ Department of Psychiatry, Samsung Medical Center, Sungkyunkwan University School of Medicine, Samsung Social Mental Health Institute, \\ Seoul, Republic of Korea \\ ${ }^{3}$ Department of Psychiatry, Inha University College of Medicine, Incheon, Republic of Korea \\ ${ }^{4}$ Department of Psychiatry \& Behavioral Science, Institute of Human Behavioral Medicine, Seoul National University College of Medicine, \\ Seoul, Republic of Korea \\ ${ }^{5}$ Department of Psychiatry, Gachon Medical School, Incheon, Republic of Korea
}

Objective The purpose of the present study was to investigate the association between Body Mass Index, suicide, perceived stress, and life dissatisfaction in a general population sample of Korean adults.

Methods A total of 6,022 nationally representative adults aged 18 to 74 were selected using a multistage cross-sectional cluster sampling method. Questionnaires regarding suicide behaviors, perceived stress, and life satisfaction were completed by the participants. They also reported their heights and weights, which were used to calculate BMI. Psychiatric disorders were diagnosed according to the Diagnostic and Statistical Manual of Mental Disorders, Fourth Edition, using the Korean version of the Composite International Diagnostic Interview.

Results The results showed that being underweight was associated with higher risk for suicide ideation [odds ratio (OR), 1.6; 95\% confidence interval $(\mathrm{Cl}), 1.18-2.05$ ] and suicide attempt (OR, 2.0, 95\% Cl, 1.23-3.31). Likewise, obesity also increased the risk of suicide ideation $(\mathrm{OR}, 1.3 ; 95 \% \mathrm{Cl}, 1.11-1.56)$ although not suicide attempt. Furthermore, underweight individuals were more likely to report severe level of perceived stress (OR, 1.7; 95\% Cl, 1.26-2.17) and life dissatisfaction (OR, 1.3; 95\% Cl, 1.07-1.68). All of the results remained significant after adjusting for age, gender, education, and psychiatric illnesses.

Conclusion This study found that being underweight is a significant risk factor for suicide and poor subjective wellbeing in Korea. It suggests that BMI status may be an important modifiable factor for improving mental health in Korea.

Psychiatry Investig 2018;15(3):272-278

Key Words Suicide, Body mass index, Perceived stress, Life dissatisfaction.

\section{INTRODUCTION}

Body mass index (BMI) is a universal measure used to determine the weight to height ratio of an individual. According to the World Health Organization (WHO), individuals with a BMI of less than 18.5 are considered to be underweight, while those with a BMI greater than 30 are classified as obese (Class

Received: February 13, 2017 Revised: April 25, 2017

Accepted: June 28, 2017

$\triangle$ Correspondence: Jin Pyo Hong, MD

Department of Psychiatry, Samsung Medical Center, Sungkyunkwan University School of Medicine, Samsung Social Mental Health Institute, 81 Irwon-ro, Gangnam-gu, Seoul 06351, Republic of Korea

Tel: +82-2-3410-3585, Fax: +82-2-3410-0500, E-mail: suhurhong@gmail.com (a) This is an Open Access article distributed under the terms of the Creative Commons Attribution Non-Commercial License (http://creativecommons.org/licenses/bync/4.0) which permits unrestricted non-commercial use, distribution, and reproduction in any medium, provided the original work is properly cited.
I obesity $\geq 30$, Class II $\geq 35$, and Class III $\geq 40$ ). A different BMI standard is recommended for Asians-as Asians in general have a lower mean or median BMI, which could lead to an underestimation of health risks. The Asian-Pacific standard considers a BMI of higher than 25 to be the marker of obesity (Class I obesity $\geq 25$, Class II $\geq 30$, and Class III $\geq 40$ ) -and a BMI of lower than 18.5 is regarded as being underweight. ${ }^{1}$

In Korea, there has been a decline in people with normal BMI over the past few years. Instead, the numbers of both obese and underweight men and women have increased. According to the National Health Insurance Service of Korea, approximately $6.0 \%$ of the population was underweight in 2010 , but the percentage increased to $7.4 \%$ in 2014 . Also, there was a $1.1 \%$ and $0.4 \%$ increase in obesity among men and women, respectively. ${ }^{2}$ Being either underweight or obese is 
known to be related to various public health problems-health issues which can pose a significant threat to our society.

Obesity is associated with a variety of different medical conditions and mortality. Diabetes, coronary artery disease, and stroke are some of the few disorders that are comorbid with obesity. ${ }^{3}$ It has also been reported that obesity has been found to have been associated with having increased psychiatric symptoms. Studies have found a strong positive relationship between obesity and lifetime diagnoses of mood disorders and anxiety disorders ${ }^{4}$ and depressive symptoms in community-based samples. ${ }^{5,6}$ In addition, obese individuals have been found to have had a higher risk for suicide as compared to individuals with a normal BMI. ${ }^{7-9}$

Similarly, a higher risk for psychiatric disorders or symptoms has been reported among underweight individuals. Sunwoo et al., ${ }^{10}$ for instance, observed that underweight Korean adults had a comparatively higher risk for depressive disorder than those with normal BMI. A 2015 study found that underweight older adults (aged over 50), followed by those with obesity, reported the highest levels of depressive symptoms in comparison to those with intermediate body weight (the data was depicted by a U-shaped association). ${ }^{11}$ An increase in suicide risk among underweight individuals has been reported by many previous studies worldwide, ${ }^{12}$ but this association has not hitherto been investigated in Korea.

So far, we have seen that many previous studies have demonstrated an association between BMI and negative indexes of life, such as psychiatric symptoms and suicide. If that is the case, it is then possible to wonder whether BMI also has a significant association with one's subjective wellbeing. Although previous studies found an association between obesity and perceived stress ${ }^{13}$ along with obesity and lower quality of life, ${ }^{14}$ its relationship with being underweight has not been investigated. We believed that the question of whether there may exist a similar association between BMI, perceived stress, and life dissatisfaction ought to be investigated. We hypothesized that such a possible association could be stronger in Korea, especially among the underweight group. It was found that Asians (including Koreans, Japanese, and Taiwanese) were more likely to see themselves as overweight even though their mean BMI were lower than other countries. Also, an attempt to lose weight was most prevalent among Asians compared to other 18 countries. ${ }^{15}$ Persistently failed efforts at weight control could increase stress and overall life dissatisfaction.

The goal of our study is to investigate the relationship between BMI and suicide behaviors (suicide ideation and attempt) using nationally representative survey data of Korean adults. The population was sampled via a stratified, multistage cluster sampling method based on the 2010 Population Census Data at the National Statistical Office of South Korea.
Furthermore, we aim to investigate whether the BMI is related to the general wellbeing of the individuals. Thus, the association between BMI, perceived stress, and life dissatisfaction will also be explored in our study.

\section{METHODS}

\section{Sample selection}

Participants of the study were recruited from the Korean Epidemiological Catchment Area Replication (KECA-R), a national survey which was conducted from July 2011 to September 2011. As indicated earlier, the population sampling of the KECA-R study used a stratified, multi-stage cluster sampling method based on the 2010 Population Census Data of the National Statistical Office (of South Korea). A total of 6,022 subjects, aged between 18 and 74, participated in the study. The response rate was $78.7 \%$. Of the 6,022 participants, 117 did not report either their weight or their height and thus had to be excluded. Thus, a total of 5,905 participants were included in this study's analysis.

\section{Assessment of suicide behaviors}

A lifetime history of suicide ideation and suicide attempt was assessed based on participants' responses. The assessment of suicide behaviors consisted of two questions which were (for suicide ideation) "Have you ever seriously thought about committing suicide?" and (for suicide attempt) "Have you ever attempted suicide?" Willing participants chose to answer either "Yes" or "No" to these questions.

\section{Assessment of perceived stress and life dissatisfaction}

All of the subjects were asked one question each about perceived stress and life dissatisfaction. For perceived stress, the question asked was "How much stress do you experience in daily life?" and for life dissatisfaction, the question was "In general, how satisfied are you with your life?"16 Each of the questions were answered according to a 5-point Likert scale. Subjects were considered to have severe perceived stress if they scored either a 1 or a 2, whereas those who scored 3 or more were counted as suffering little to no stress. Similarly, subjects with either a score of 1 or 2 were classified as life dissatisfaction group. The rest of the participants with scores greater than 2 were considered to have a personally satisfying life. For both of the questions, there were only few.

\section{Assessment of BMI}

The BMI for each person was calculated from the self-reported weight and height of each. Using the WHO's BMI criteria for the Asian-Pacific region, participants were divided into four subgroups: underweight $\left(<18.5 \mathrm{~kg} / \mathrm{m}^{2}\right)$, normal (18.5-22.95 
$\left.\mathrm{kg} / \mathrm{m}^{2}\right)$, overweight $\left(23-24.9 \mathrm{~kg} / \mathrm{m}^{2}\right)$, or obese $\left(\geq 25 \mathrm{~kg} / \mathrm{m}^{2}\right)$.

\section{Assessment of psychiatric disorders}

All of the participants were interviewed face-to-face, using the Korean version of the Composite International Diagnostic Interview (K-CIDI). ${ }^{17}$ The K-CIDI allows for diagnosis of various psychiatric disorders based on the criteria of the fourth Diagnostic and Statistical Manual of Mental Disorders (DSMIV). K-CIDI interviewers received a 5-day training session based on standard protocols and training materials developed by the WHO. ${ }^{18}$ A lifetime prevalence of mood, anxiety, and alcohol disorders were examined in this article.

\section{Ethics statement}

The present study protocol was reviewed and approved by the Institutional Review Board of Seoul National University (IRB No. C-1104-092-359). Informed consent was submitted by all subjects when they were enrolled.

\section{Statistical analysis}

As stated above, the subjects were divided into four BMI groups-underweight, normal, overweight or obese. The group differences in demographic variables were investigated using a chi-square test or Analysis of Variance (ANOVA). Bivariate logistic regression models were used to examine the relationship between BMI and dependent variables, which were: Suicide behaviors (ideation and attempt), perceived stress, and life dissatisfaction. The normal BMI group was regarded as the reference group. The analyses adjusted for the potential compounding effects of age, gender, marital status, education, monthly income, and psychiatric disorders. SPSS 21.0 (IBM Corp., Armonk, NY, USA) was used for all statistical analyses, and the statistical significance was tested at an alpha level of $<0.05$.

\section{RESULTS}

\section{Demographic characteristics of the sample}

Each of the participants was classified into one of the four BMI groups. Out of the total number of participants, $5.8 \%$

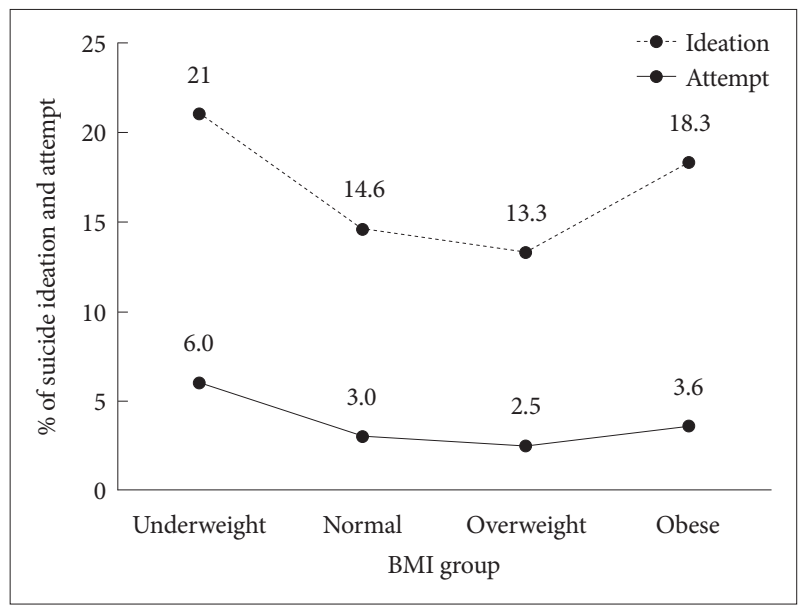

Figure 1. Prevalence of suicide ideation and suicide attempt in the BMI groups. BMI: Body Mass Index.

Table 1. Demographic and clinical characteristics of the study sample by the BMI groups

\begin{tabular}{|c|c|c|c|c|c|}
\hline \multirow{2}{*}{ Characteristics } & \multicolumn{4}{|c|}{ BMI groups } & \multirow{2}{*}{$\mathrm{p}$ value } \\
\hline & $<18.5$ (underweight) & $18.5-22.9$ (normal) & 23-24.9 (overweight) & $\geq 25$ (obese) & \\
\hline No. of participants & 343 & 2,884 & 1,357 & 1,321 & \\
\hline Age (mean, SD) & $35.4(14.9)$ & $41.2(14.8)$ & $46.4(13.9)$ & $45.0(13.7)$ & $<0.0001$ \\
\hline Female gender $(\%)$ & 79.6 & 57.4 & 41.8 & 36.2 & $<0.0001$ \\
\hline Marital status (\%) & & & & & $<0.0001$ \\
\hline Married & 39.1 & 57.4 & 68.3 & 68.5 & \\
\hline Divorced/widowed/separated & 10.2 & 11.2 & 12.7 & 12.7 & \\
\hline Unmarried & 50.7 & 31.4 & 18.9 & 18.8 & \\
\hline Education years (mean, SD) & $12.8(3.6)$ & $12.7(4.9)$ & $12.0(3.9)$ & $12.2(5.7)$ & $<0.0001$ \\
\hline Monthly household income (\%) & & & & & 0.01 \\
\hline$<2000 \$$ & 42.7 & 37.5 & 37.1 & 40.7 & \\
\hline $2,000-3,000 \$$ & 23.8 & 26.8 & 27.2 & 26.9 & \\
\hline$\geq 3,000 \$$ & 33.5 & 35.7 & 35.7 & 32.4 & \\
\hline \multicolumn{6}{|l|}{ Psychiatry disorders (\%) } \\
\hline Mood disorders & 9.3 & 7.6 & 5.5 & 8.5 & 0.01 \\
\hline Anxiety disorders & 10.7 & 9.1 & 8.5 & 7.7 & 0.27 \\
\hline Alcohol disorders & 7.9 & 12.6 & 15.6 & 15.0 & $<0.05$ \\
\hline
\end{tabular}

BMI: Body Mass Index 




Figure 2. Prevalence of severe perceived stress and life dissatisfaction in BMI groups. BMI: Body Mass Index. were underweight $\left(<18.5 \mathrm{~kg} / \mathrm{m}^{2}\right), 48.8 \%$ were normal $(18.5-$ $\left.22.9 \mathrm{~kg} / \mathrm{m}^{2}\right), 23.0 \%$ were overweight (23-24.9 kg/m²), and $22.4 \%$ were obese $\left(\geq 25 \mathrm{~kg} / \mathrm{m}^{2}\right)$. The demographic and clinical characteristics of each BMI group are shown in Table 1. Age, gender, marital status, education, and prevalence of alcohol disorders showed significant group differences. Each BMI group's risk of suicidal behaviors, perceived severe stress, and life dissatisfaction are shown in Figures 1 and 2.

\section{BMI, suicide ideation, and suicide attempt}

Table 2 provides the unadjusted and adjusted odd ratios (Model I and Model II) for suicide ideation and suicide attempt for each BMI group. Compared to individuals with a normal BMI, the underweight group (OR, 1.6; 95\% CI, 1.182.05) and obese group (OR, 1.3; 95\% CI, 1.11-1.56) showed significantly greater odds for suicide ideation. The significance persisted after adjusting for age, gender, marital status, educa-

Table 2. Adjusted ORs of suicide ideation and suicide attempt by BMI groups (relative to normal BMI group)

\begin{tabular}{|c|c|c|c|c|c|c|}
\hline \multirow{3}{*}{ Variables } & \multicolumn{6}{|c|}{$\mathrm{BMI}\left(\mathrm{kg} / \mathrm{m}^{2}\right)$} \\
\hline & \multicolumn{2}{|c|}{$<18.5$ (underweight) } & \multicolumn{2}{|c|}{ 23-24.9 (overweight) } & \multicolumn{2}{|c|}{$\leq 25$ (obese) } \\
\hline & OR & $95 \% \mathrm{CI}$ & OR & $95 \% \mathrm{CI}$ & OR & $95 \% \mathrm{CI}$ \\
\hline \multicolumn{7}{|l|}{ Unadjusted OR } \\
\hline Suicide ideation & 1.6 & $1.18-2.05^{* *}$ & 0.9 & $0.74-1.08$ & 1.3 & $1.11-1.56^{* *}$ \\
\hline Suicide attempt & 2.0 & $1.23-3.31^{*}$ & 0.8 & $0.54-1.21$ & 1.2 & $0.83-1.70$ \\
\hline \multicolumn{7}{|l|}{ Model I ${ }^{\mathrm{a}}$} \\
\hline Suicide ideation & 1.8 & $0.99-1.90$ & 1.0 & $0.79-1.20$ & 1.3 & $1.08-1.61^{* *}$ \\
\hline Suicide attempt & 1.8 & $1.04-3.18^{*}$ & 0.8 & $0.52-1.38$ & 1.2 & $0.76-1.77$ \\
\hline \multicolumn{7}{|l|}{ Model II' } \\
\hline Suicide ideation & 1.6 & $1.10-2.21^{*}$ & 0.8 & $0.77-1.21$ & 1.3 & $1.06-1.62 *$ \\
\hline Suicide attempt & 2.4 & $1.34-4.37^{* *}$ & 0.8 & $0.48-1.28$ & 1.2 & $0.78-1.89$ \\
\hline
\end{tabular}

adjusted for gender, age, marital status, education and monthly income, badjusted for gender, age, marital status, education, monthly income and psychiatric disorders (mood disorders, anxiety disorders, alcohol use disorders). ${ }^{*} \mathrm{p}<0.05,{ }^{* *} \mathrm{p}<0.01$. BMI: Body Mass Index, OR: odd ratio

Table 3. Adjusted ORs of severe perceived stress, life dissatisfaction by BMI status (relative to normal BMI group)

\begin{tabular}{|c|c|c|c|c|c|c|}
\hline \multirow{3}{*}{ Variables } & \multicolumn{6}{|c|}{$\mathrm{BMI}\left(\mathrm{kg} / \mathrm{m}^{2}\right)$} \\
\hline & \multicolumn{2}{|c|}{$<18.5$ (underweight) } & \multicolumn{2}{|c|}{ 23-24.9 (overweight) } & \multicolumn{2}{|c|}{$\leq 25$ (obese) } \\
\hline & OR & $95 \% \mathrm{CI}$ & OR & $95 \% \mathrm{CI}$ & OR & $95 \% \mathrm{CI}$ \\
\hline \multicolumn{7}{|l|}{ Unadjusted OR } \\
\hline Severe perceived stress & 1.7 & $1.26-2.17^{* * *}$ & 0.9 & $0.73-1.1$ & 1.06 & $0.88-1.29$ \\
\hline Life dissatisfaction & 1.3 & $1.07-1.68^{*}$ & 1.0 & $0.86-1.14$ & 1.0 & $0.89-1.16$ \\
\hline \multicolumn{7}{|l|}{ Model I ${ }^{\mathrm{a}}$} \\
\hline Severe perceived stress & 1.4 & $1.00-1.90^{*}$ & 0.9 & $0.74-1.12$ & 1.0 & $0.78-1.18$ \\
\hline Life dissatisfaction & 1.3 & $1.03-1.74^{*}$ & 0.9 & $0.79-1.06$ & 0.9 & $0.81-1.09$ \\
\hline \multicolumn{7}{|l|}{ Model II ${ }^{\mathrm{b}}$} \\
\hline Severe perceived stress & 1.5 & $1.04-2.02^{*}$ & 0.9 & $0.76-1.12$ & 0.9 & $0.76-1.16$ \\
\hline Life dissatisfaction & 1.4 & $1.06-1.80^{* *}$ & 0.9 & $0.79-1.06$ & 0.9 & $0.80-1.08$ \\
\hline
\end{tabular}

adjusted for gender, age, marital status, education and monthly income, badjusted for gender, age, marital status, education, monthly income and psychiatric disorders (mood disorders, anxiety disorders, alcohol use disorders). ${ }^{*} \mathrm{p}<0.05,{ }^{* *} \mathrm{p}<0.01,{ }^{* * *} \mathrm{p}<0.001$. BMI: Body Mass Index, OR: odd ratio 
tion, monthly income, and psychiatric disorders. The underweight group had a significantly higher risk of having attempted suicide (AOR, 2.4; 95\% CI, 1.34-4.37) but obesity had no significant association with attempting suicide (Table 2).

\section{BMI, severe perceived stress, and life dissatisfaction}

Table 3 provides unadjusted and adjusted odd ratios for severe perceived stress and life dissatisfaction. Relative to individuals with a normal BMI, underweight individuals were more likely to have severe perceived stress (OR, 1.7; 95\% CI, 1.26-2.17) and life dissatisfaction (OR, 1.3; 95\% CI, 1.071.68). Adjustment for the potentially confounding factors of age, gender, marital status, education, monthly income, and psychiatric disorders did not have an impact on the significance of the result. Other BMI groups had no significant association with severe perceived stress and life dissatisfaction (Table 3).

\section{DISCUSSION}

In this paper, we have shown that among the adult population in Korea, underweight individuals had an elevated risk for having attempted suicide. Underweight individuals in our study were twice more likely than their normative counterparts to have attempted suicide. Consistent with our study, a systematic review of BMI and suicidal death demonstrated an inverse relationship between the two, meaning that underweight individuals have the greatest risk of suicide, whereas obese people have the lowest risk. ${ }^{19}$ The result could partially be related to the high prevalence of depressive disorder among the underweight Korean adult population, given that depressive individuals are more vulnerable to suicide. ${ }^{10}$ However, the associations between being underweight and attempting suicide remained significant even after controlling for mood disorders as well as for anxiety and alcohol use disorders. This suggests that the correlation between BMI and suicide cannot by fully explained by psychiatric disorders. Some see biological mechanisms as playing a role in linking BMI and suicide. For example, a high level of tryptophan and serotonin among individuals with a higher BMI could reduce suicide risk. ${ }^{20}$ Also, insulin resistance in obese individuals increases brain serotonin level, which may decrease suicide behaviors. ${ }^{21}$ However, these explanations-in their focus on obesity and low suicide risk-do not fully explain why underweight individuals are at higher risk of suicide. Future research should try to better understand the suicidal tendencies of underweight individuals.

Another important result of our study was the finding of a higher risk for suicide ideation among obese and underweight subjects. This suggests that deviation from the normal BMI range is a significant risk factor for suicide ideation. The increase in prevalence of suicide ideation among obese individuals is most often interpreted as being due to a decrease in selfesteem due to social stigma. In Korea, there is a pervasive societal norm that mastery over one's body is a symbol of selfmanagement and competence. We believe that such societal pressure, along with one's own desire for a thinner body, can cause psychological distress to those who are fatter or thinner than others. Wardle et al. ${ }^{15}$ found that the majority of Korean women (77\%) were trying to lose weight. This percentage was the highest in comparison to 22 other countries. The pressure to lose weight can lead to engagement in inappropriate weight control behaviors such as fasting or purging/ vomiting. Recent study found that individuals with high inappropriate weight control behaviors were more likely to have suicide ideation or attempted suicide. ${ }^{22}$

Contrary to our findings with regards to suicide ideation and BMI, a systemic review by Perera et al. ${ }^{19}$ found little evidence to support an association between suicide ideation and $\mathrm{BMI}$, after adjusting for the potentially confounding variables. The results of our study remained significant even after adjusting for such confounding variables as age, gender, marital status, education, monthly income, and psychiatric disorders. Our study focused on a nationally representative sample of more than 5,000 Korean adults-a data set which we believe to be of sufficient reliability from which to draw conclusions about BMI and suicide among Koreans. Also, our study involved face-to-face interviewing using K-CIDI to diagnose possible psychiatric disorders and its effect on the results. A majority of the BMI and suicide ideation studies included in the literature review did not adjust for psychiatric disorders. These factors could result in findings inconsistent with those of previous studies which have examined suicide ideation and BMI.

Underweight individuals were not the only ones who were found to have a higher risk for suicidal behaviors. The subjective well-being of underweight individuals seemed to be lower than that of other BMI groups. Individuals who are underweight perceived themselves to be suffering from severe stress, and they were also more likely to express dissatisfaction with their life. Although our study did not examine why underweight individuals have lower subjective well-being, several hypotheses can be made. The underweight group in our study consisted of mostly females (nearly $80 \%$ ) who are younger and single, possibly with higher sensitivity to their body size. The females were more likely to believe that they are overweight, ${ }^{15}$ and their perception of being overweight was associated with poorer life satisfaction. ${ }^{23}$ In other words, underweight individuals in our study were found to believe that they are overweight (although physically, they are actually 
not), and perhaps a consequence of this is their poorer life satisfaction. Another explanation for the severe stress and life dissatisfaction of the underweight group could be related to a shared personality trait such as neuroticism. A number of existing studies found a high level of neuroticism among underweight individual $\mathrm{s}^{24,25}$ and among those with anorexia nervosa. ${ }^{26,27}$ Neuroticism was also associated with a lower level of happiness. ${ }^{28,29}$ Our study did not consider the potentially confounding variables of either subjective perception of body size or personality traits, so future studies should examine each when investigating one's psychological wellbeing.

Our study had several limitations. First, our research used self-reported body weights and heights to calculate BMI. The use of an objective measure could have been more accurate, but many previous researches have also used BMI based on self-reported measures. ${ }^{7,10}$ Second, our study did not analyze the results for males and females separately. Nearly $80 \%$ of the participants in the underweight group were female, so it was difficult to run separate tests for each gender. Instead, we have treated gender as a confounding variable to reduce its impact on the result. Gender and other sociodemographic variables did not have a significant impact on our results. Third, the relationship between life satisfaction and suicide can be influenced by severity of depressive symptoms but we only adjusted the effects of depressive disorders. Hence effects of minor depressive symptoms could have affected the results. Fourth, the results of our study do not explain why underweight individuals were more vulnerable to suicide behaviors, severe stress, or life dissatisfaction. Future research should explore possible mechanisms behind the relationship, along with possible mediating variables. Furthermore, the design of our study did not allow us to examine causal inferences between variables. The results of our study do not provide direction of effect.

Despite its limitations, our study has important strengths. It is the first study to investigate the relationship between BMI and suicidal behaviors among a nationally representative general population in Korea. BMI and suicide attempt were found to be related according to an L-shape curve, while prevalence of suicide ideation presented according to a U-shaped curve. Similarly, the relationship observed for severe perceived stress and life dissatisfaction was L-shaped. Examination of stress and life dissatisfaction in relation to BMI enabled us to understand that being underweight is a risk factor-not only for a serious psychopathology like suicidal behavior, but also for subjective well-being. The findings carry the implication that it is important to consider a person's BMI status when examining suicidal tendencies and subjective wellbeing.

\section{Acknowledgements}

This work was supported by a research grant from the Korean Ministry of Health and Welfare and Korea Healthcare Technology R\&D project, Ministry of Health and Welfare, Republic of Korea [Grant number HI12C0035].

The authors thank all the interviewers and the Korean Ministry of Health and Welfare for their cooperation and assistance.

\section{REFERENCES}

1. Weisell RC. Body Mass Index as an indicator of obesity. Asia Pacific J Clin Nur 2002;11:S681-S684.

2. National Health Insurance Service. National Health Insurance Statistical Year book. Available at: http://www.nhis.or.kr/menu/boardRetriveMenuSet.xx?menuId=F3321. Accessed September 22, 2016.

3. Kopelman P. Health risks associated with overweight and obesity. Obes Rev 2007;8:13-17.

4. Simon GE, Von Korff M, Saunders K, Miglioretti DL, Crane PK, van Belle $\mathrm{G}$, et al. Association between obesity and psychiatric disorders in the US adult population. Arch Gen Psychiatry 2006;63:824-830.

5. de Wit L, Luppino F, van Straten A, Penninx B, Zitman F, Cuijpers P. Depression and obesity: a meta-analysis of community-based studies. Psychiatry Res 2010;178:230-235.

6. Faith MS, Butryn M, Wadden TA, Fabricatore A, Nguyen AM, Heymsfield SB. Evidence for prospective associations among depression and obesity in population-based studies. Obes Rev 2011;12:e438-e453.

7. Carpenter KM, Hasin DS, Allison DB, Faith MS. Relationships between obesity and DSM-IV major depressive disorder, suicide ideation, and suicide attempts: results from a general population study. Am J Public Health 2000;90:251-257.

8. Dong C, Li WD, Li D, Price RA. Extreme obesity is associated with attempted suicides: results from a family study. Int J Obes Relat Metab Disord 2005;30:388-390.

9. Lester D, Iliceto P, Pompili M, Girardi P. Depression and suicidality in obese patients. Psychol Rep 2011;108:367-368.

10. Sunwoo YK, Bae JN, Hahm BJ, Lee DW, Park JI, Cho SJ, et al. Relationships of mental disorders and weight status in the Korean adult population. J Korean Med Sci 2011;26:108-115.

11. Noh JW, Kwon YD, Park J, Kim J. Body mass index and depressive symptoms in middle aged and older adults. BMC Public Health 2015; $15: 310$.

12. Klinitzke G, Steinig J, Bluher M, Kersting A, Wagner B. Obesity and suicide risk in adults--a systematic review. J Affect Disord 2013;145:277284.

13. Matuska K, Bass J. Life balance and stress in adults with medical conditions or obesity. OTJR (Thorofare N J) 2016;36:74-81.

14. Strine TW, Chapman DP, Balluz LS, Moriarty DG, Mokdad AH. The Associations Between Life Satisfaction and Health-related Quality of Life, Chronic Illness, and Health Behaviors among U.S. Communitydwelling Adults. J Community Health 2008;33:40-50.

15. Wardle J, Haase AM, Steptoe A. Body image and weight control in young adults: international comparisons in university students from 22 countries. Int J Obes 2005;30:644-651.

16. Kobau R, Sniezek J, Zack MM, Lucas RE, Burns A. Well-being assessment: an evaluation of well-being scales for public health and population estimates of well-being among US adults. Appl Psychol Health Wellbeing 2010;2:272-297.

17. Cho MJ, Hahm BJ, Bae JN, Suh T, Lee DW, Cho SJ. Development of the Korean version of Composite International Diagnostic Interview (K-CIDI). J Korean Neuropsychiatr Assoc 2002;41:1015-4817.

18. World Health Organization. CIDI, Core version 2.1 Trainer's Manual. Geneva: World Health Organization, 1997, p.1-244.

19. Perera S, Eisen RB, Dennis BB, Bawor M, Bhatt M, Bhatnagar N, et al. Body Mass Index is an important predictor for suicide: results from a systematic review and meta-analysis. Suicide Life Threat Behav 2016; 46:697-736. 
20. Mann JJ, Currier DM. Stress, genetics and epigenetic effects on the neurobiology of suicidal behavior and depression. Eur Psychiatry 2010; 25:268-271

21. Golomb BA, Tenkanen L, Alikoski T, Niskanen T, Manninen V, Huttunen $\mathrm{M}$, et al. Insulin sensitivity markers: predictors of accidents and suicides in Helsinki Heart Study screenees. J Clin Epidemiol 2002;55: 767-773.

22. Lee SA, Jang SY, Shin J, Ju YJ, Nam JY, Park EC. The association between inappropriate weight control behaviors and suicide ideation and attempt among Korean adolescents. J Korean Med Sci 2016;31:15291537.

23. Herman KM, Hopman WM, Rosenberg MW. Self-rated health and life satisfaction among Canadian adults: associations of perceived weight status versus BMI. Qual Life Res 2013;22:2693-2705.

24. Terracciano A, Sutin AR, McCrae RR, Deiana B, Ferrucci L, Schlessinger
D, et al. Facets of personality linked to underweight and overweight. Psychosom Med 2009;71:682-689.

25. Kakizaki M, Kuriyama S, Sato Y, Shimazu T, Matsuda-Ohmori K, Nakaya $\mathrm{N}$, et al. Personality and body mass index: a cross-sectional analysis from the Miyagi Cohort Study. J Psychosom Res 2008;64:71-80.

26. Cassin SE, von Ranson KM. Personality and eating disorders: a decade in review. Clin Psychol Rev 2005;25:895-916.

27. Bulik CM, Sullivan PF, Tozzi F, Furberg H, Lichtenstein P, Pedersen NL. Prevalence, heritability, and prospective risk factors for anorexia nervosa. Arch Gen Psychiatry 2006;63:305-312.

28. Lu L. The relationship between subjective well-being and psychosocial variables in Taiwan. J Soc Psychol 1995;135:351-357.

29. Vittersø J, Nilsen F. The conceptual and relational structure of subjective well-being, neuroticism, and extraversion: once again, neuroticism is the important predictor of happiness. Soc Indic Res 2002;57:89-118. 